\title{
Nifedipine prevents hepatic fibrosis in a non-alcoholic steatohepatitis model induced by an L-methionine-and choline-deficient diet
}

\author{
HIRONORI NAKAGAMI ${ }^{1}$, MUNEHISA SHIMAMURA ${ }^{1}$, TAKASHI MIYAKE ${ }^{1}$, TAKASHI SHIMOSATO ${ }^{2}$, \\ NORIKO MINOBE ${ }^{2}$, TOSHINORI MORITANI ${ }^{2}$, MARIANA KIOMY OSAKO ${ }^{3,4}$, FUTOSHI NAKAGAMI ${ }^{4}$, \\ HIROSHI KORIYAMA ${ }^{4}$, MARIKO KYUTOKU ${ }^{4}$, HIDEO SHIMIZU ${ }^{3,4}$, \\ TOMOHIRO KATSUYA ${ }^{4}$ and RYUICHI MORISHITA ${ }^{4}$ \\ ${ }^{1}$ Division of Vascular Medicine and Epigenetics, Osaka University United Graduate School of Child Development, \\ Suita, Osaka 565-0871; ${ }^{2}$ Research Department, Nissei Bilis Co. Ltd., Koka, Shiga 528-0052; \\ Departments of ${ }^{3}$ Geriatric Medicine and ${ }^{4}$ Clinical Gene Therapy, Osaka University \\ Graduate School of Medicine, Suita, Osaka 565-0871, Japan
}

Received April 13, 2011; Accepted August 8, 2011

DOI: $10.3892 / \mathrm{mmr} .2011 .594$

\begin{abstract}
Recent reports have shown that nifedipine, a calcium channel blocker, increases peroxisome proliferator-activated receptor- $\gamma$ (PPAR $\gamma$ ) activity. Since PPAR $\gamma$ agonists, such as pioglitazone and rosiglitazone, are effective in reducing non-alcoholic steatohepatitis (NASH) and cirrhosis in animal models, we examined the protective effects of nifedipine, as compared with bezafibrate, a PPAR $\alpha$ agonist, in a NASH model induced by an L-methionine- and choline-deficient (MCD) diet. An MCD diet for 20 weeks changed the color of the rat liver to yellow with an irregular surface, whereas the color of the liver in both the bezafibrate and nifedipine treatment groups was markedly changed to yellow-brown with a smooth surface. Furthermore, nifedipine, as well as bezafibrate, significantly prevented liver fibrosis induced by an MCD diet, as assessed by Masson's trichrome staining, accompanied by a significant decrease in serum AST. Overall, nifedipine treatment resulted in an improvement in NASH, similar to bezafibrate, in a rat model. In hypertensive patients with metabolic syndrome, nifedipine may provide additional benefits, beyond its blood pressure-lowering effects, to prevent NASH and fatty liver disease.
\end{abstract}

Correspondence to: Professor Ryuichi Morishita, Department of Clinical Gene Therapy, Osaka University Graduate School of Medicine, 2-2 Yamada-oka, Suita, Osaka 565-0871, Japan

E-mail: morishit@cgt.med.osaka-u.ac.jp

Key words: calcium channel blocker, peroxisome proliferatoractivated receptor- $\gamma$, non-alcoholic steatohepatitis, hepatic fibrosis

\section{Introduction}

Non-alcoholic steatohepatitis (NASH) recently has become the most common liver disease in the world, affecting approximately $20 \%$ of obese individuals (1-3). NASH is strongly associated with insulin resistance and the metabolic syndrome and is believed to progress to cirrhosis (4). Recent studies suggest that non-alcoholic fatty liver disease (NAFLD) is an independent cardiovascular risk factor that increases cardiovascular mortality $(5,6)$ and is also associated with endothelial dysfunction as an initial stage of atherosclerosis (7). A 'two-hit' theory has been proposed for the progression of NAFLD to NASH, and hepatic steatosis associated with metabolic syndromes could be the first hit, followed by a second insult from increased oxidative stress, leading to fibrosis (8). However, there are no approved treatments for NASH. Recently, the PIVENS trial demonstrated an improvement in NASH histology with either vitamin E or an insulin-sensitizing peroxisome proliferator-activated receptor- $\gamma$ (PPAR $\gamma$ ) ligand (9). Consistently, rosiglitazone was also found to attenuate the histological findings and decreased elevated serum enzyme levels in a NASH mouse model (10). In addition, a PPAR $\alpha$ agonist, bezafibrate, is known to prevent NASH in animal models (11).

The most relevant risk factor for NAFLD is the presence of metabolic syndrome, which includes insulin resistance, diabetes, obesity, dyslipidemia and hypertension $(1,3,4)$. Thus, it is important to explore potential drug therapies to prevent the progression of NAFLD to NASH using the drugs already employed to treat such conditions. Among these numerous drugs, including antihypertensive, antidiabetic and antihyperlipidemic drugs, the present study focused on nifedipine, a calcium channel blocker that is widely used to treat hypertensive patients as several recent reports have demonstrated that nifedipine activates PPAR $\gamma$ activity in different cell types (12-14). Here, we demonstrated the preventive action of 
A

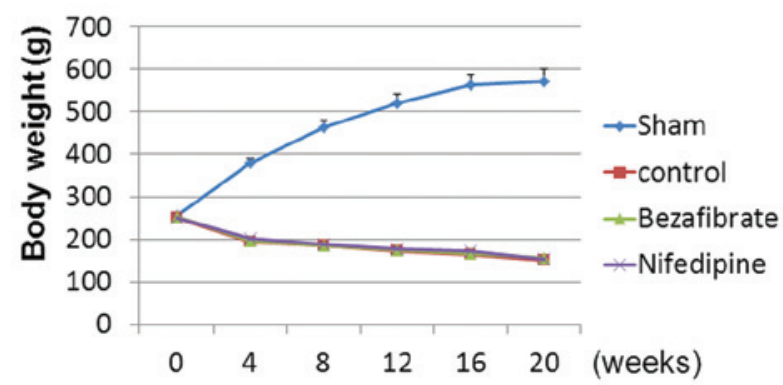

B

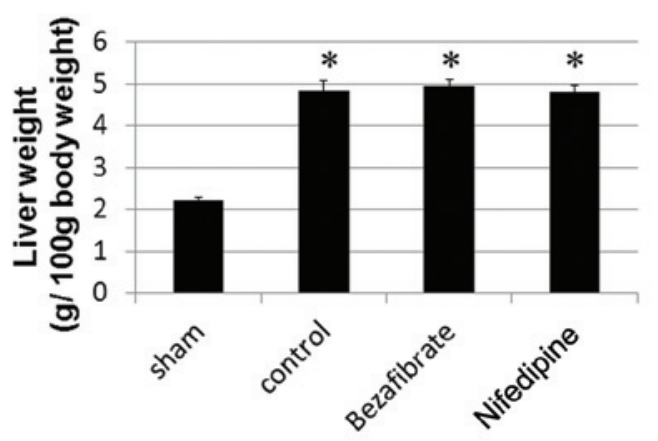

Figure 1. Body weight and liver weight of rats fed an MCD diet for 20 weeks. (A) Body weight in each group at $0,4,8,12,16$ and 20 weeks after an MCD diet. (B) Liver weight at 20 weeks after an MCD diet (g/100 g body weight). Groups: sham, fed a control diet $(n=6)$; MCD, fed an MCD diet $(n=7)$; bezafibrate, fed an MCD diet and co-administered bezafibrate $(n=6)$; and nifedipine, fed an MCD diet and co-administered nifedipine $(n=6)$. ${ }^{*} \mathrm{P}<0.05$ as compared with the 'sham' group.

nifedipine on NASH, similar to bezafibrate, in a rat model using a methionine- and choline-deficient (MCD) diet.

\section{Materials and methods}

Animals and diets. The experiments were approved by the Ethics Committee for Animal Experiments of Osaka University, Graduate School of Medicine. Male Wistar rats were obtained from Jackson Laboratory (Bar Harbor, ME). Rats (aged 8 weeks) were fed a control diet (no. 518811, Dyets Inc.) or an MCD diet (no. 518810, Dyets Inc.) to induce hepatic fibrosis as a result of overloading with endogenous triglycerides (15). Briefly, the rats were divided into four groups: i) a group fed a control diet (sham, $\mathrm{n}=6$ ); ii) a group fed an MCD diet (control: MCA, $\mathrm{n}=6$ ); iii) a group fed an MCD diet and orally co-administered bizafibrate $10 \mathrm{mg} / \mathrm{kg} /$ day (bezafibrate: MCD + bezafibrate, $n=7$ ); and iv) a group fed an MCD diet and orally co-administered nifedipine $10 \mathrm{mg} / \mathrm{kg} /$ day (nifedipine: MCD + nifedipine, $\mathrm{n}=7$ ). Nifedipine was provided by Bayer Yakuhin Ltd. and bezafibrate was obtained from Sigma-Aldrich (St. Louis, MO).

Rats had free access to water and food during the experimental periods. Body weight was measured every week, and liver weight, serum AST (GOT) and total protein were measured for 20 weeks after the start of the MCD diet.

Evaluation of liver fibrosis. Extracted liver samples were photographed, and the liver weight was measured after the
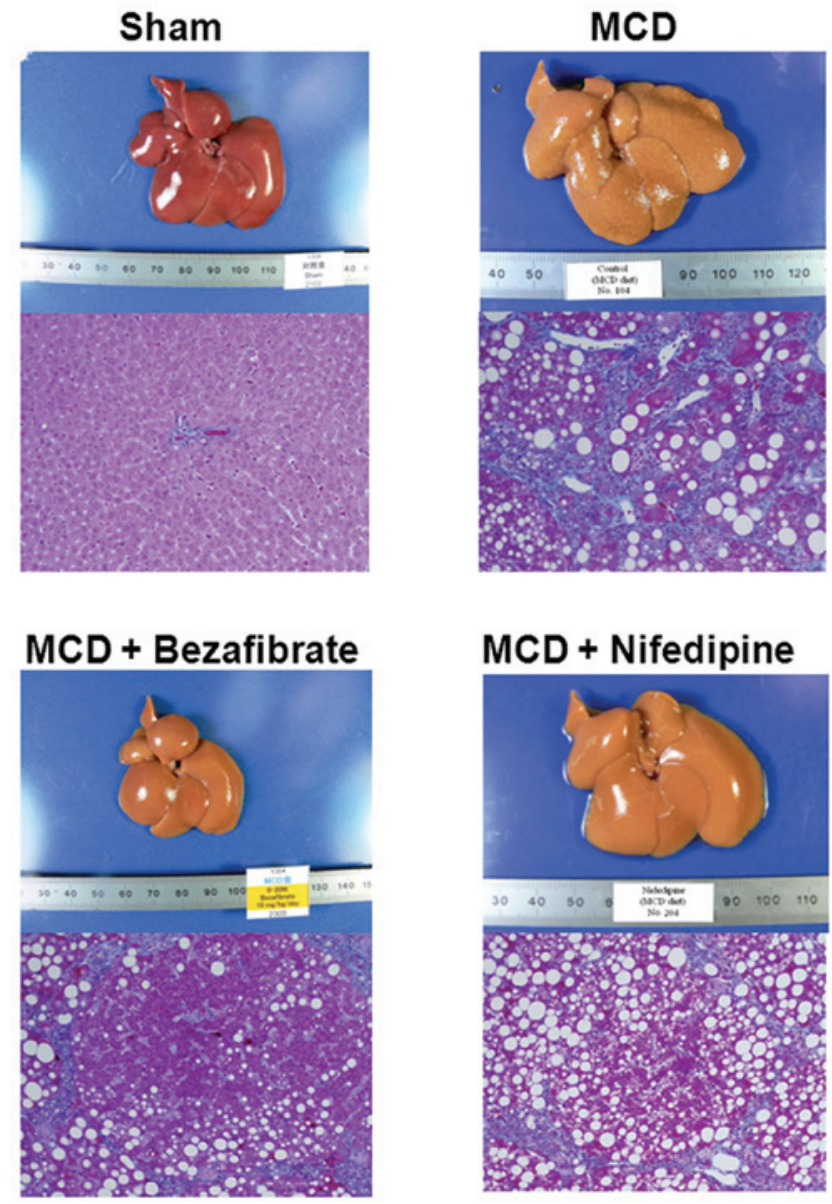

Figure 2. Representative images of a rat liver in an MCD diet-induced NASH model after 20 weeks. The upper panel shows representative images of a liver and the lower panel shows images of Masson's trichrome staining in a section of the liver. Groups: sham, fed a control diet $(\mathrm{n}=6)$; MCD, fed an MCD diet $(\mathrm{n}=7) ; \mathrm{MCD}+$ bezafibrate, fed an MCD diet and co-administered bezafibrate $(\mathrm{n}=6)$; and MCD + nifedipine, fed an MCD diet and co-administered nifedipine $(n=6)$.

removal of water and the attached tissues. Liver findings were evaluated by two independent researchers in a double-blind manner with regard to discoloration, rough surface and degree of nodules. Half of the liver tissue was fixed in $10 \%$ formaldehyde, and each slide was stained with H\&E or Masson's trichrome staining. The area of fibrosis was evaluated by positive staining with Masson's trichrome staining, measured using Win Roof (version 5.5, Mitsuya-Shoji) and displayed as the percentage of the total area (16).

Statistical analysis. All values are expressed as the mean \pm SD. Data were compared using ANOVA, followed by Dunnett's test for pair-wise comparisons against 'control' and by Turkey's test for multiple comparisons. All statistical analyses were performed using Stat-View 5.0 software (SAS Institute, Inc., $\mathrm{NC})$. Values of $\mathrm{P}<0.05$ were considered statistically significant.

\section{Results}

The body weight of rats fed the control diet was significantly increased during the experimental period, whereas rats fed the 
A

\begin{tabular}{lcll} 
& Discoloration & Surface & Nodule \\
\hline Sham & Brown & Smooth & None \\
Control & Yellow & Irregular & Diffuse \\
Bezafibrate & $\begin{array}{c}\text { Yellowish } \\
\text {-brown }\end{array}$ & Not smooth & Local \\
Nifedipine & $\begin{array}{c}\text { Yellowish } \\
\text {-brown }\end{array}$ & Not smooth & Local \\
& & &
\end{tabular}

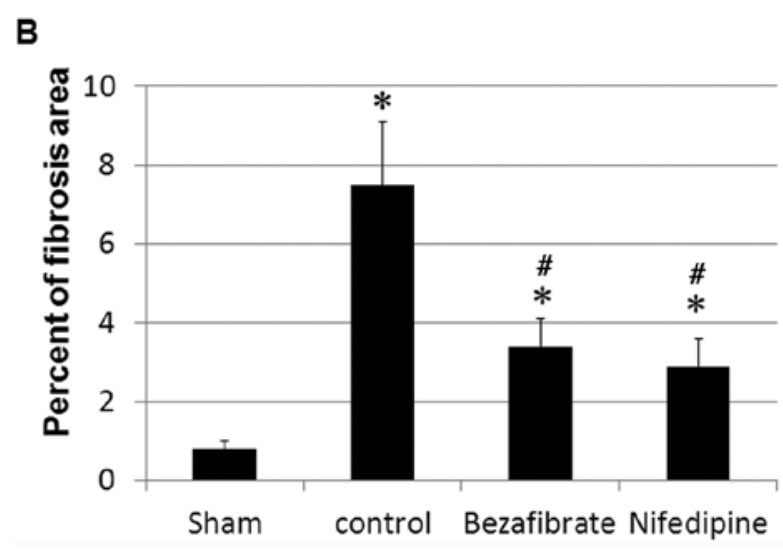

Figure 3. Evaluation of the external liver and quantification of the fibrotic area. (A) The external liver was evaluated in terms of discoloration (brown, yellow-brown or yellow), rough surface (smooth, not smooth or irregular), and degree of nodules (none, local or diffuse). (B) The fibrosis area was quantified by calculating the positive area (blue color) with Masson's trichrome staining. Groups: sham, fed a control diet $(n=6)$; MCD, fed an MCD diet $(n=7)$; MCD + bezafibrate, fed an MCD diet and co-administered bezafibrate $(n=6)$; and $\mathrm{MCD}+$ nifedipine, fed an MCD diet and co-administered nifedipine $(\mathrm{n}=6)$. ${ }^{\text {*}} \mathrm{P}<0.05$ compared with the 'sham' group; ${ }^{*} \mathrm{P}<0.05$ compared with the 'control'.
A

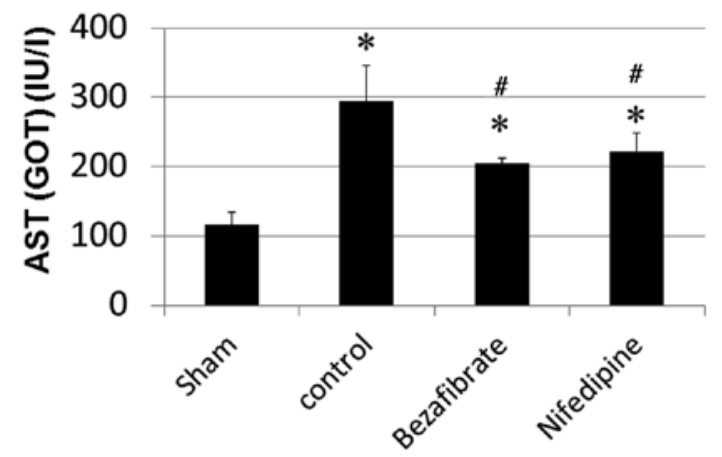

B

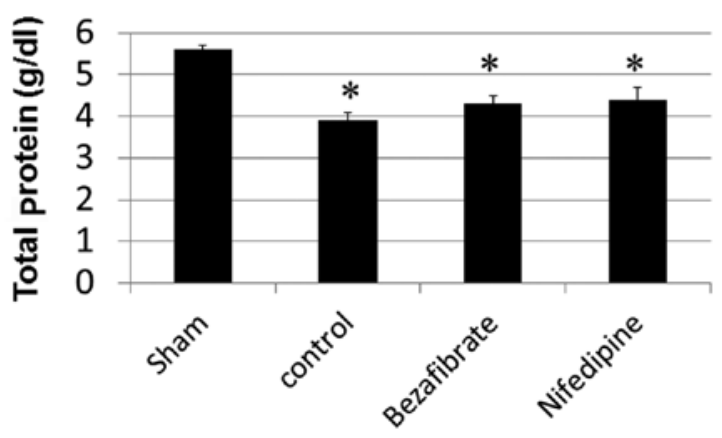

Figure 4. Serum AST (GOT) and total protein levels at 20 weeks in an MCD diet-induced NASH model. (A) Serum AST (GOT) (IU/l) and (B) total protein $(\mathrm{g} / \mathrm{dl})$ at 20 weeks after an MCD diet. Groups: sham, fed a control diet $(\mathrm{n}=6) ; \mathrm{MCD}$, fed an MCD diet (n=7); MCD + bezafibrate, fed an MCD diet and co-administered bezafibrate $(\mathrm{n}=6)$; and $\mathrm{MCD}+$ nifedipine, fed an MCD diet and co-administered nifedipine $(\mathrm{n}=6)$. $\mathrm{P}<0.05$ compared with the 'sham' group; " $\mathrm{P}<0.05$ compared with the 'control'.
MCD diet for 20 weeks had a significantly decreased body weight (Fig. 1A, P<0.01). In contrast, liver weight as a fraction of body weight was significantly increased in the MCD diet group as compared with the sham group (Fig. $1 \mathrm{~B}, \mathrm{P}<0.01$ ), due to the accumulation of triglycerides in the liver in this model. Although nifedipine treatment did not change the body weight and liver weight as compared with vehicle, the surface of the liver was markedly changed by nifedipine treatment. In control rats fed the MCD diet, we observed that the color of the liver changed to yellow, with an irregular surface and diffuse nodules (Fig. 2). However, the liver color was still brown and its surface was smooth in the rats treated with nifedipine, similar to bezafibrate (Figs. 2 and 3A). In the evaluation of liver fibrosis by Masson's trichrome staining, the MCD diet markedly induced liver fibrosis, while the administration of nifedipine, as well as bezafibrate, significantly attenuated the progression of liver fibrosis (Figs. 2 and 3A). Moreover, a significant increase in serum AST (GOT) levels and a decrease in total protein were observed in control rats fed the MCD diet for 20 weeks, suggesting liver dysfunction. As shown in Fig. 4A, the serum AST level was significantly decreased by nifedipine, whereas the administration of bezafibrate also decreased the MCD-induced AST elevation $(\mathrm{P}<0.01)$. The administration of bezafibrate, as well as nifedipine, resulted in a tendency toward a decrease in serum total protein levels.

\section{Discussion}

Metabolic syndrome patients present with various abnormalities, such as hypertension, insulin resistance and hypertriglycemia, which are independent risk factors for cardiovascular events. Recently, it has become known that metabolic syndrome is strongly associated with the development and progression of NAFLD (17). Currently, NAFLD, as well as NASH, are considered independent risk factors for cardiovascular events (6). Therefore, preventing the progression of NAFLD to NASH in metabolic syndrome patients has become an important therapeutic option for treating metabolic syndrome. However, there are very limited options to prevent NASH and NAFLD at this point. One potential group of drugs, the PPAR $\gamma$ ligands, such as rosiglitazone and pioglitazone, is reported to prevent NASH in animal models and in human patients $(9,10,15)$. PPAR $\gamma$ ligands decrease steatosis through the inhibition of the activation of inflammatory and fibrotic genes, probably due to a decrease in oxidative stress. However, as PPAR $\gamma$ ligands are antidiabetes drugs, only diabetic patients can use them. Therefore, it is important to explore the potential therapeutic tools to prevent NASH. Among the drugs for common adult diseases, we focused on nifedipine as an antihypertensive drug and bezafibrate as an antihypertriglycemia drug as both drugs are widely used in clinical medicine. 
In the present study, we demonstrated that the administration of nifedipine, as well as bezafibrate, improved the liver dysfunction and fibrosis induced by an MCD diet. Consistent with previous reports $(15,18)$, MCD diet-fed rats exhibited a marked accumulation of triglycerides in the liver due to the inhibition of lipid transportation by decreased phosphatidylcholine, which leads to inflammation and fibrosis in the liver. In an NAFLD mouse model of the metabolic syndrome, bezafibrate caused an increase in antioxidants ( $\alpha$-tocopherol concentrations and paraoxonase activity) and decreases in glutathione peroxidase and glutathione reductase activity, accompanied by a decrease in macrovesicular steatosis, an increase in hepatic phospholipids, and decreases in hepatic triglycerides and cholesterol (11). Based on the two-hit theory for the progression of NAFLD to NASH, the principal second hit could be oxidative stress. Thus, the antioxidant effects of PPAR $\alpha$ and PPAR $\gamma$ agonists may also be effective in the NASH model.

In contrast, nifedipine, an L-type calcium channel blocker, is known to have various favorable pleiotropic effects in several animal models, such as atherosclerosis, aortic aneurysmal formation and diabetic nephropathy models $(12,13,19)$. Notably, one of the mechanisms of these effects would be mediation by PPAR $\gamma$ activation. Ishii et al demonstrated that nifedipine activated PPAR $\gamma$ through the inactivation of ERK, leading to the suppression of MCP-1 and ABCA1 (ATP-binding cassette transporter, subfamily A, member 1) expression in macrophages. Nifedipine has also been reported to increase luciferase activity by full-length PPAR $\gamma$ but not by a PPAR $\gamma$-GAL4 chimera, indicating that nifedipine is not a direct PPAR $\gamma$ ligand and may not increase intracellular natural PPAR $\gamma$ ligands, whereas nifedipine has an agonistic effect on PPAR $\gamma$ activity (13). Notably, amlodipine did not increase PPAR $\gamma$ activity, indicating that PPAR $\gamma$ activation is not a common effect of L-type calcium channel blockers. In addition, nifedipine was found to enhance the effects of other PPAR $\gamma$ agonists, pioglitazone and ciglitazone-induced PPAR $\gamma$ activation (13). Thus, the combination of nifedipine with thiazolidinediones may enhance the prevention of NAFLD or NASH in metabolic syndrome patients.

Overall, nifedipine, as well as bezafibrate, demonstrated potential action in improving NASH induced by an MCD diet. Drugs for hypertension and hypertriglyceridemia (nifedipine or bezafibrate) may be useful in preventing NASH in metabolic syndrome patients, although further studies are necessary.

\section{Acknowledgements}

We thank Ms. Hizuki Hamada and Natsuki Yasumasa for their kind assistance in all experiments.

\section{References}

1. Marchesini G, Bugianesi E, Forlani G, et al: Non-alcoholic fatty liver, steatohepatitis, and the metabolic syndrome. Hepatology 37: 917-923, 2003.

2. Sanyal AJ: AGA technical review on non-alcoholic fatty liver disease. Gastroenterology 123: 1705-1725, 2002.

3. Angulo P: Non-alcoholic fatty liver disease. N Engl J Med 346: 1221-1231, 2002.

4. Chitturi S, Abeygunasekera S, Farrell GC, et al: NASH and insulin resistance: insulin hypersecretion and specific association with the insulin resistance syndrome. Hepatology 35: 373-379, 2002.

5. Vuppalanchi R and Chalasani N: Non-alcoholic fatty liver disease and non-alcoholic steatohepatitis: selected practical issues in their evaluation and management. Hepatology 49: 306-317, 2009.

6. Hamaguchi M, Kojima T, Takeda N, et al: Non-alcoholic fatty liver disease is a novel predictor of cardiovascular disease. World J Gastroenterol 13: 1579-1584, 2007.

7. Villanova N, Moscatiello S, Ramilli S, et al: Endothelial dysfunction and cardiovascular risk profile in non-alcoholic fatty liver disease. Hepatology 42: 473-480, 2005.

8. Browning JD and Horton JD: Molecular mediators of hepatic steatosis and liver injury. J Clin Invest 114: 147-152, 2004.

9. Sanyal AJ, Chalasani N, Kowdley KV, et al: Pioglitazone, vitamin E, or placebo for non-alcoholic steatohepatitis. N Engl J Med 362: 1675-1685, 2010.

10. Gupte AA, Liu JZ, Ren Y, et al: Rosiglitazone attenuates age- and diet-associated non-alcoholic steatohepatitis in male low-density lipoprotein receptor knockout mice. Hepatology 52: 2001-2011, 2010.

11. Ackerman Z, Oron-Herman M, Rosenthal T, et al: Effects of amlodipine, captopril, and bezafibrate on oxidative milieu in rats with fatty liver. Dig Dis Sci 53: 777-784, 2008.

12. Matsui T, Yamagishi S, Takeuchi M, Ueda S, Fukami K and Okuda S: Nifedipine, a calcium channel blocker, inhibits advanced glycation end product (AGE)-elicited mesangial cell damage by suppressing AGE receptor (RAGE) expression via peroxisome proliferator-activated receptor-gamma activation. Biochem Biophys Res Commun 385: 269-272, 2009.

13. Ishii N, Matsumura T, Kinoshita H, et al: Nifedipine induces peroxisome proliferator-activated receptor-gamma activation in macrophages and suppresses the progression of atherosclerosis in apolipoprotein E-deficient mice. Arterioscler Thromb Vasc Biol 30: 1598-1605, 2010.

14. Iwai M, Kanno H, Inaba S, et al: Nifedipine, a calcium-channel blocker, attenuated glucose intolerance and white adipose tissue dysfunction in type 2 diabetic KK-A(y) mice. Am J Hypertens 24: 169-174, 2011.

15. Rinella ME, Elias MS, Smolak RR, Fu T, Borensztajn J and Green RM: Mechanisms of hepatic steatosis in mice fed a lipogenic methionine choline-deficient diet. J Lipid Res 49: 1068-1076, 2008.

16. Nakagami H, Kiomy Osako M, Nakagami F, et al: Prevention and regression of non-alcoholic steatohepatitis (NASH) in a rat model by metabosartan, telmisartan. Int J Mol Med 26: 477-481, 2010.

17. Hamaguchi M, Kojima T, Takeda N, et al: The metabolic syndrome as a predictor of non-alcoholic fatty liver disease. Ann Intern Med 143: 722-728, 2005.

18. Rinella ME and Green RM: The methionine-choline deficient dietary model of steatohepatitis does not exhibit insulin resistance. J Hepatol 40: 47-51, 2004.

19. Tomita N, Yamasaki K, Izawa K, et al: Inhibition of experimental abdominal aortic aneurysm progression by nifedipine. Int J Mol Med 21: 239-244, 2008. 